\title{
An Improved Specimen Preparation of Porous Powder Materials for Transmission Electron Microscopy
}

\author{
Na Yeon Kim, Gyeong Hee Ryu, Hyo Ju Park and Zonghoon Lee
}

School of Materials Science and Engineering, UNIST (Ulsan National Institute of Science and Technology), Ulsan, S. Korea

There are many methods to prepare TEM specimens such as Focused ion beam (FIB) milling, dimpling, ion milling, and so forth according to the kinds of samples and the purpose of observation. In case of porous materials, it is difficult to ma ke TEM specimen due to low stability of boundaries between vacuum and materials. As research has actively done on secondary lithium ion batteries, the demands for research on microstructure of cathode materials has been increased because microstructural changes can be a basis for the degradation of capacity. [1] However, most of cathode materials are composed of aggregated few micrometer-sized grains, so they have many micro-sized pores and grain boundaries due to the weak adhesion between grains during the synthesis process. They also can be expanded according to the increase of the number of cycling of charge and discharge because of the volume shrinkage caused by oxygen loss.[2]

Figure 1(a) shows a SEM image of cross sectional TEM specimen for cathode material after 300 cycling at $60^{\circ} \mathrm{C}$ prepared by FIB. There are many pores between grains whose sizes are a few micrometers. The purpose of TEM study for cathode materials is mainly to observe the microstructural changes especially at edge state of grains because the degradation of capacity can be explained in accordance with the degree of the change. But many of edge position can be damaged due to the presence of micro-sized pores which are initial milling points by ion beam of FIB. Figure 1(b) is a HAADF image to show the contaminated edge position by ion beam of FIB. Therefore, it is not appropriate to investigate the effect of microstructural change on capacity degradation on this specimen.

To avoid the contamination, new specimen preparation method can be introduced before FIB milling process. Figure 2(a) shows the sketchy design. This method is based on specimen preparation methods before dimpling process of powder and sandwich sample. [3] Epoxy is used to paste the substrates or adhere the powders as being cured at a specific temperature. In this method, the mixture of powder and epoxy is input between the substrates, not in copper ring and after that, process of pressing the substrates is introduced for epoxy to permeate the pores and grain boundaries much more and to remove the extra epoxy, which can raise the density of materials. In other words, high temperature to set the epoxy and high pressure to improve the adhesion and density can improve the permeation of epoxy, so epoxy can fill the pores and grain boundaries whose sizes are from hundreds of nanometer to few micrometer as shown in Figure 2(b). So the epoxy filler takes a role of protective coat of edge state from ion beam of FIB. Figure 2(c) is a HRTEM image of the microstructure of cathode materials. It shows low damaged edge state and microstructure well, so it can used to investigate the effect of microstructural changes on degradation of capacity. This method not only improve the quality of TEM specimen, but also increase the efficiency of specimen preparation as decreasing the time for specimen preparation about one-fifth and increasing the probability to acquire specimens having the desired thickness and sizes.

References

[1] Muto, S., et al., Journal of The Electrochemical Society, 2009. 156(5): A371 
[2] Zheng, S., et al., Journal of The Electrochemical Society, 2011. 158(4): A357

[3] David B. W., et al., Transmission electron microscopy, Springer, New York, 2009.

[4] This work was supported by the IT R\&D Program of MKE/KEIT [10041856, Technology

development for life improvement of high Ni composition cathode at high temperature $\left.\left(>60^{\circ} \mathrm{C}\right)\right]$.
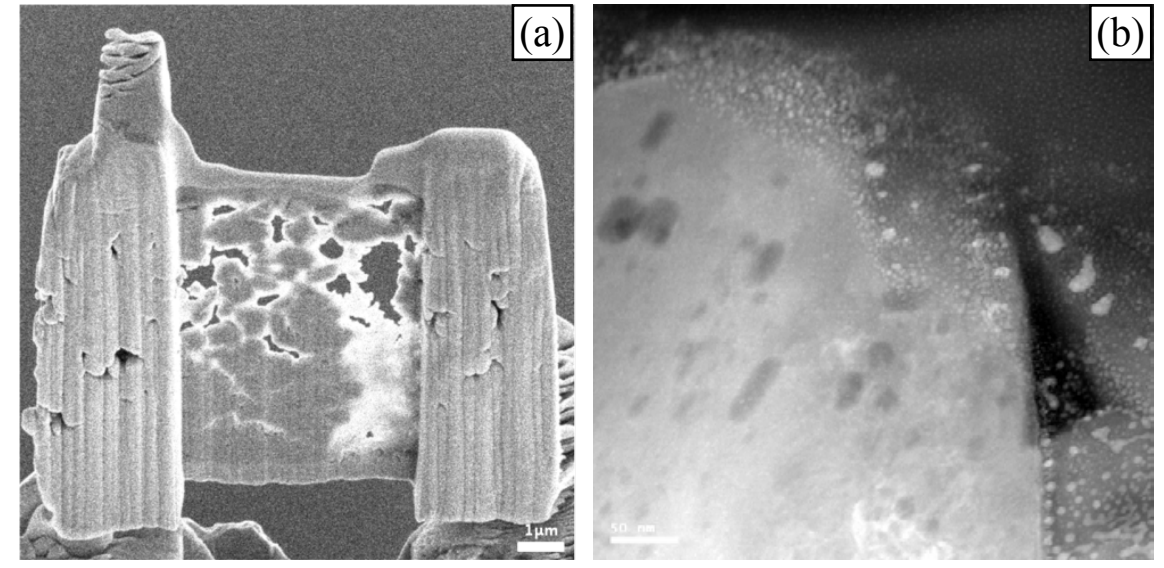

Figure 1. Damaged micro-porous cathode materials prepared by ion beam in FIB. (a) SEM image when captured at preparing TEM specimen with FIB which composed of nickel, cobalt, and manganese after 300 cycling at $60^{\circ} \mathrm{C}$. There are many pores due to the high number of cycling. (b) HAADF image showing the contaminated edge state where is the critical region for TEM observation.
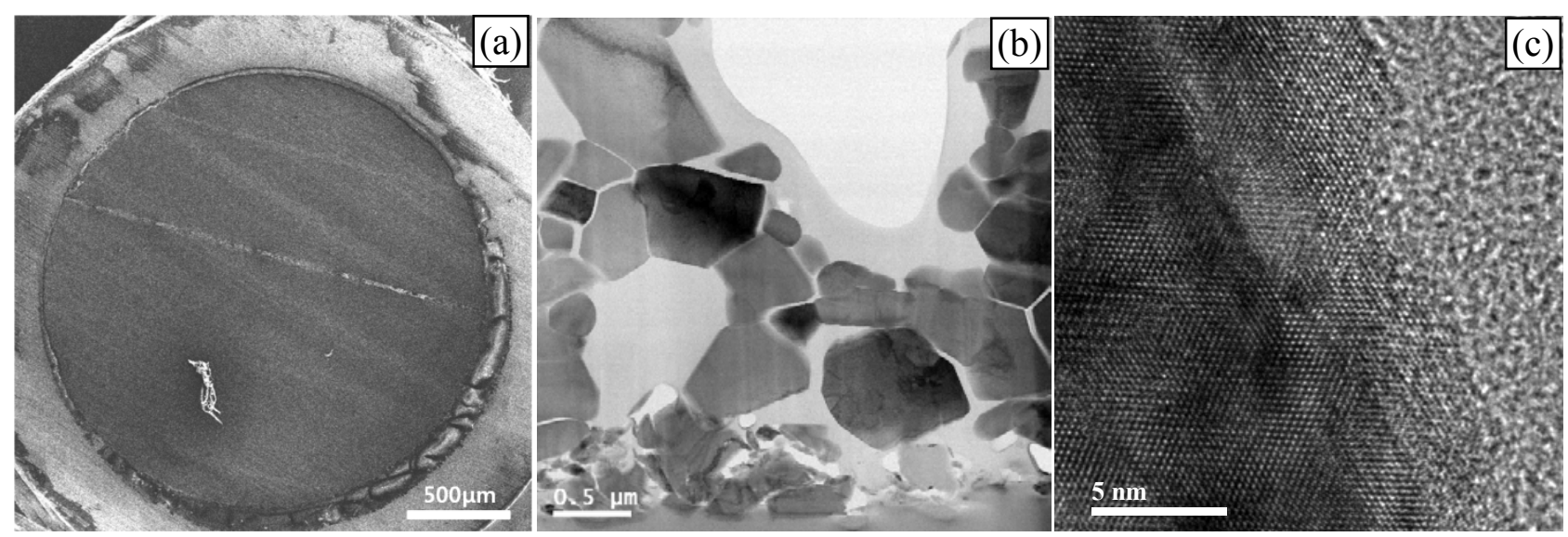

Figure 2. Low contaminated TEM specimen by FIB with new sampling method (a) SEM image showing the design of new sampling method before FIB milling process. The brighter and thick layer between substrates contains the cathode materials as powder morphology. (b)Low magnification BF image showing that the epoxy fills pores and grain boundaries whose sizes are from hundreds of nanometer to few micrometer, so it takes a protective role, (c) URTEM image showing fine edge state protected by epoxy. 